\title{
Influence of tolerances on error estimation in P3R and 4R planar mechanisms
}

\author{
Ankur Jaiswal $^{1}$ · H. P. Jawale ${ }^{2}$
}

Received: 10 July 2020 / Accepted: 21 December 2021 / Published online: 28 January 2022

(c) The Author(s) 2022

\begin{abstract}
Four-bar linkages form a primitive configuration of many mechanisms. The output of these mechanisms deviates from the desired one due to many factors including tolerance on links. For satisfactory application, the mechanism performance needs to be characterized. This article presents a treatment on the synthesis and analysis of linear-input P3R (1-Prismatic, 3-Revolute) and rotary-input 4R (4-Revolute) planar mechanism configurations, and the estimation of mechanical error under the influence of link tolerances. A detailed methodology is presented for error estimation as part of performance analysis for each mechanism. As an illustrative case, mechanisms are considered to operate under identical output generation conditions and comparative performance evaluation is carried out. Effect of link proportion is studied to investigate the mechanism behavior related to mechanical error. Comparison of error provides the basis for selecting the mechanism that gives better performance. The proposed methodology demonstrates generalized characterization of the mechanical error sources with validation.
\end{abstract}

Keywords Planar mechanism $\cdot$ Link tolerances $\cdot$ Mechanical error $\cdot$ Comparison of error $\cdot$ Validation

\section{List of symbols}

$l_{1}, l_{2}, l_{3}$ and $l_{4}$

$\theta_{1}, \theta_{3}$

$A, B, C, D, E, F, G, H$ and I

$\Delta l_{1}, \Delta l_{2}, \Delta l_{3}$ and $\Delta l_{4}$
Link Lengths: input link, coupler link, output link and fixed link, respectively Angular displacement at input and output link, respectively

Coefficient of the design parameters

Deviation of the link lengths link 1 to 4 , respectively

$$
E_{m 1}, E_{m 2}, E_{m 3} \text { and } E_{m 4}
$$

$\Psi$

$l_{t 1}, l_{t 2}, l_{t 3}$ and $l_{t 4}$

K

$E_{d 1}, E_{d 2}, E_{d 3}$ and $E_{d 4}$
Total mechanical error on link 1, link 2, link 3 and Link 4 , respectively, by modified Taylor series approximation Internal angle between Link 1 and Link 4

Total link Lengths on link 1 to 4 after deviations Proportion factor Total mechanical error on link 1, link 2, link 3 and Link 4 , respectively, by geometrical method
Technical Editor: Victor Juliano De Negri.

Ankur Jaiswal

ankurmech13@gmail.com

H. P. Jawale

hpjawale@rediffmail.com

1 Department of Mechatronics, Manipal Institute of Technology, Manipal Academy of Higher Education, (MAHE), Manipal 576104, Karnataka, India

2 Department of Mechanical Engineering, Visvesvaraya National Institute of Technology, Nagpur 440010, India

\section{Introduction}

Four-bar linkages are commonly used in machines and mechanisms for achieving the desired path, function and motion generation task. Four bar mechanisms and its inversion are used in variety of applications as in biomedical equipment, automobiles, machine linkages, etc. The revolute and prismatic joint configurations are used as a part of planar and parallel manipulators. Various complex mechanisms are studied with four-link chain as primitive configurations. 
The performance characteristics, namely positioning accuracy, structural and mechanical errors, depend on the kind of input condition of the mechanism [1,2]. These mechanisms employ rotary or linear actuators for desired task. The selection of particular actuator is governed by functional requirements such as input range, position, velocity and accelerations of output link as well as force required at output link. Therefore, comparative performance analysis is important for selection of linear or rotary actuation. The primary performance features for such analysis are the positional error due to the structural and mechanical parameters. The structural error is the inability in reaching the desired function or position due to the link dimension after dimensional synthesis of a specific configuration. For few precision positions, the structural error becomes zero. The mechanical error occurs due to the deviations of link dimensions from the designed dimensions on account of design constraints, manufacturing process limitations and subsequent assembly. The factors affecting the performance of manipulators include clearance, backlash, drive error, assembly error, deflection and expansion of linkages, etc., found in the literature. As link tolerances are inevitable, the mechanism does have positional deviations. The exact tolerance after design and manufacturing is possible to quantify and thus it is possible to estimate mechanical error. The attempt is presented herewith to investigate the mechanical error due to quantified link tolerances in general.

Many researchers have performed structural and mechanical error analysis for path, motion and function generation problems in four-link mechanisms [3-6]. Dubowsky et al. and Erkaya et al. [7] discussed the effect of clearance and link flexibility on stresses in joints of high speed and slidercrank mechanisms under dynamic conditions. Sharfi and Smith [8] investigated the link-length tolerance and joint clearance in the multi-link mechanism. K. L Ting et al. [9] presented modeling and analysis of joint clearances and resulting uncertainty in revolute as well as prismatic pairs in multi-loop mechanisms. Flores [10] established a general methodology for quantifying the effects of kinematic position errors due to manufacturing and assembly tolerance for open as well as closed chain planar mechanisms. Ting, et al. described an approach to determine the effects of joint clearance on position and orientation deviation in linkages, based on N-bar rotatability laws concept and evolutionary optimization techniques [11]. The problems of positional error, performance quality, transmission angle of open and closed loop mechanisms, using various techniques such as stochastic approach [3, 12-14], probabilistic model [15], loop closure method [16], genetic algorithm [17] are found to be employed in the literature. The probability approach is used to determine the uncertainty effect of link tolerance and joint clearance in serial, planar and spatial robots $[18,19]$. Zhang and Xianmin [20] and Chen, et al. [21] studied the influence of clearance in joints of planar 3-RRR and 4-RRR mechanisms, wherein comparison between the two mechanisms based on position deviation is presented. Jawale and Thorat [22] analyzed the 4R, 2-serial, and P3R mechanisms and influence of clearances and backlash is presented. Tian et al. and Flores et al. [23-26] investigated the dynamics of multi-body mechanical systems by analytical, numerical and experimental techniques and considered imperfect clearance, friction, link flexibility and geometric imperfections effects of clearances in lubricated joints. Zhan et al. applied a hybrid method of first-order second moment (FOSM) technique for multiple uncertainty in planar parallel manipulator like manufacturing tolerances, input errors and joint clearances, while carrying out motion reliability analysis [27]. Erkaya discussed the effect of joint clearances in robotic system and kinematic and dynamic analysis of trajectory of endeffector is carried out to know the motion sensitivity [28]. Cammarata and Erkaya investigated the dynamic behavior in a 3D slider-crank mechanism and RRR spherical parallel manipulator under the effect of with and without clearance joints using computational and elastostatic approach, respectively [29]. The effect of clearance sizes and driving speeds results are determined and verified by ADAMS simulations software [30]. Erkaya proposed the ANFIS approach algorithm to optimize the trajectory parameters of a walking mechanism with joint clearance and compared the obtained results by analytical approach [31].

Erkaya et al. evaluated kinematic and dynamic performance analysis of the four-bar and slider joint mechanism under effect of joint clearance $[32,33]$. Tsai and Lai evaluated kinematic position and accuracy analysis of the multilink mechanism using wrench screw method. The obtained results with joint clearances are compared with that of the ideal mechanism [34]. Jawale and Thorat analyzed the position accuracy of the serial and closed chain manipulator [35]. Li et al. presented model for angular errors due to joint clearances in multi-loop structures. By using optimization method and geometric method, the angular errors are determined. The results were verified using Monte Carlo simulations [36]. Tsai and Lai analyzed the position errors and transmission quality of the mechanism affects due to joint clearances [37]. Wu and Rao applied the interval approach for the modeling of tolerances and clearances and the fuzzy error analysis of mechanisms. Further, comparison of the interval number approach and traditional approach is presented out [38]. Zhang and Han proposed a method based on reliability which considers the randomness of the link lengths and estimates the error within a sphere of radius equal to the estimated error through saddle point approximation [39].

Jaiswal and Jawale et.al evaluated mechanical error under the influence of line tolerance in four bar revolute joint mechanisms $[40,41]$. The extensive work presented in the 
literature was found to cater the effect on characteristic of mechanism output.

The effect of tolerances, specifically on revolute joint (4R) mechanisms, is seen to be considered in most of the literature cited above. The effect of clearance contributes to randomness in the behavior of the mechanism, whereas tolerances are presumed to give cumulative effect on mechanism performance. In mechanical design and manufacturing sciences, tolerances on machine components are stacked giving cumulative end values. In closed chain mechanisms, it leads to mechanical error but the rule is not established. The influence of tolerance needs to be investigated in mechanisms. The attempt is made herewith to uncover the variation in error pattern under the influence of link known tolerance. The mechanisms providing limited range of motion are of special use in automation application. The task of position generation, function generation and rigid body guidance are accomplished through use of mechanisms by applying reversible rotary actuation or linear actuation. The drivespecific behavior of the mechanism needs to be analyzed for performance features like mechanical error under the influence of link tolerance. The error estimation carried out by authors Jaiswal and Jawale [40, 41] is extended further to four bar prismatic input (P3R) mechanism. Investigation of effect of link proportions on error pattern through generalized formulation is also aimed in the work presented herewith. Further, comparative performance evaluation is targeted which may lead to select a configuration for a given application. The extent of sensitivity to the deviations on account of tolerances is the aim of present work. The present work considers the exact tolerance on the links, which is important for estimating the mechanical error rather than range of the error at the coupler positions, which use the probabilistic analysis of dimensional deviations on the links to a specified problem threshold. The traditional reliability-based methods are complex and thus are insufficient to consider randomness in link length variation or mechanical error estimation.

The major limitations of such methods lie in estimation of the exact error for continuous trajectory. The proposed method in this paper enables to estimate mechanical error at any set of finite coupler positions which is important for performance analysis over a trajectory.

The work presented in this paper is organized as follows-Sect. 2 deals with methodology and Sect. 3 deals with kinematic synthesis and mechanical error formulation of $4 \mathrm{R}$ and P3R mechanisms. Section 4 deals with formulations for exact error prediction for both configurations. Section 5 presents concept of identical operating condition for possible comparative study, with demonstration of sample case. Also, considerations for various link proportions are dealt in this section. Section 6 provides results and discussions followed by comparative error analysis for both mechanism configurations in Sect. 7. Conclusions and future scope are discussed in Sect. 8.

\section{Methodology}

The P3R and 4R mechanism are investigated for mechanical error herewith using traditional approximate estimation method at initial treatment. Error propagation method is well known statistical technique based on first-order Taylor's series approximation for error analysis. The literature reports this method being used for mechanical error analysis; with probable approximate estimations. This method with small error in terms of known link deviation takes into account input variables, leading to a prediction of maximum and total deviation. From the literature, it is found that the effect of clearances and deviations on link lengths does not have straightforward correlation with the positional error in case of closed chain mechanisms; anticipating the error to vary with change in local actuation specific input variables. This is the motive behind not considering the input as in Gaussian distribution but to use small error in terms of known link deviation.

The exact error estimation method is also tailored herewith, which involves known fixed values or constraints that are used to find an exact solution. This method is used with known independent variable (tolerances in this case) and the final value of deviations is obtained. The analytical treatment gives an exact and pointed deviation. This is the strength of this technique, suitable for analyzing the mechanical error in individual configuration. The deviations of all links in mechanisms are precisely carried on to contribute in the final estimation.

For each mechanism, synthesis equation is developed, and a mechanical error equation is obtained by considering link tolerance as variable for possible drive inputs (i.e., rotary and linear actuation) for approximation and exact estimation, respectively.

Effect of link proportions on error is evaluated. An equivalence relation between the inputs under identical output conditions is established for both mechanisms by analytical and CAD approaches. The effect of link tolerance is analyzed as specific error to account for theoretical and actual conditions. Finally, the performance of the two mechanisms with rotary and linear actuations is compared.

The methodology applied for analyzing the error is described in flowchart (Fig. 1). The formulation was developed for a manipulator analysis through approximate approach and exact approach. The validation of the results for these methods is carried out through CAD model. The deviations in end effector position under the influence of link tolerance are estimated through both methods on $4 \mathrm{R}$ and P3R configurations, in order to obtain link proportions. Later on, effect of coupler 


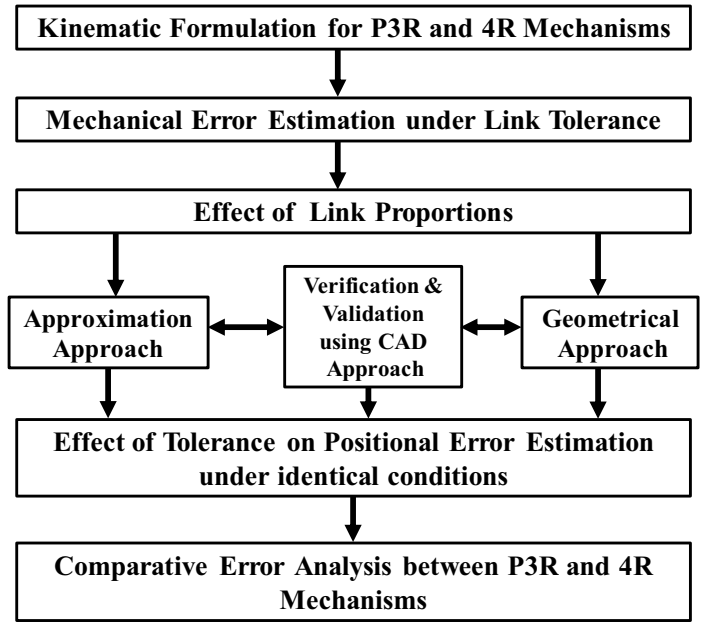

Fig. 1 Flowchart

position on error is estimated. Comparison of the error for $4 \mathrm{R}$ and P3R mechanisms is carried out.

\section{Kinematic synthesis of $4 R$ and P3R mechanism}

\subsection{Characteristic equation-4R mechanism}

Consider a 4R mechanism in a rectangular coordinate system as shown in Fig. 2. Taking design parameters $l_{1}, l_{2}, l_{3}, l_{4}, \theta_{1}$, as inputs and $\theta_{3}$ as the output $[1,41]$, the derivation of displacement equations is written as,

For point $A$ :

$x_{2}=l_{1} \cdot \cos \theta_{1}$

$y_{2}=l_{1} \cdot \sin \theta_{1}$

For point $B$ :

$x_{3}=-l_{4}+l_{3} \cdot \cos \theta_{3}$

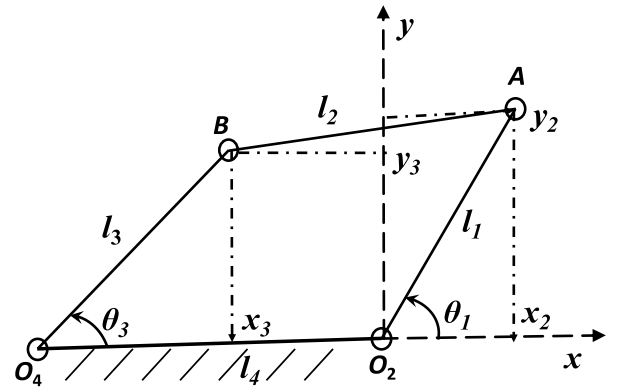

Fig. 2 4R mechanism configuration $y_{3}=l_{3} \cdot \sin \theta_{3}$

The distance between the point $A$ and point $B$ is fixed and equal to $l_{2}$

$\left(x_{2}-x_{3}\right)^{2}+\left(y_{2}-y_{3}\right)^{2}=l_{2}^{2}$

Substituting Eqs. (1) to (4) in Eq. (5),

$\left(l_{1} \cos \theta_{1}+l_{4}-l_{3} \cos \theta_{3}\right)^{2}+\left(l_{1} \sin \theta_{1}-l_{3} \sin \theta_{3}\right)^{2}=l_{2}^{2}$

On simplifying Eq. (6) in trigonometric form,

$A \sin \theta_{3}+B \cos \theta_{3}=C$

$A=\sin \theta_{1}$

$B=\frac{l_{4}}{l_{1}}+\cos \theta_{1}$

$C=\frac{l_{4}}{l_{3}} \cos \theta_{1}+\frac{l_{1}^{2}-l_{2}^{2}+l_{3}^{2}+l_{4}^{2}}{2 l_{1} l_{3}}$

$\tan \frac{\theta_{3}}{2}=\frac{A \pm \sqrt{A^{2}+B^{2}-C^{2}}}{B+C}$

Equation (11) is used to obtain the output link angle of the $4 \mathrm{R}$ mechanism.

\subsection{Characteristic equation-P3R mechanism}

Consider a planar four-link mechanism with a linear actuation (P3R) as shown in Fig. 3. The equation can be obtained from position analysis of the configuration to determine the input and output angles due to varying input displacement of linear drive motor or actuator. Let instantaneous leg length $v$ be given as

$v=\left(l_{1}+l_{2}\right)+\delta$

where, $\delta=$ displacement of actuator.

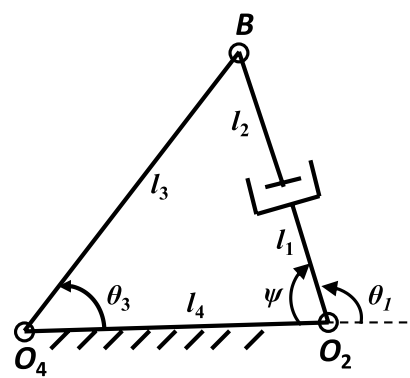

Fig. 3 P3R mechanism configuration 
Using cosine theorem,

$\psi=a \cos \left(\frac{v^{2}+l_{4}^{2}-l_{3}^{2}}{2 l_{4} v}\right)$

$\theta_{3}=a \cos \left(\frac{l_{3}^{2}+l_{4}^{2}-v^{2}}{2 l_{3} l_{4}}\right)$

$\theta_{1}=180-\psi$

Equation (14) is used to determine the output link angle of the P3R mechanism.

\subsection{Mechanical error-approximation approach}

Consider a 4R planar mechanism with constant length parameters $l_{1}, l_{2}, l_{3}, l_{4}$ transforming a motion defined by an input variable $\left(\theta_{1}\right)$ and output variable $\left(\theta_{3}\right)$. The primary condition of loop closure for existence of four-bar mechanism may be written in general form as

$f\left(l_{1}, l_{2}, l_{3}, l_{4}, \ldots, \theta_{i}, \theta_{o}\right)=0$

Each independent parameter inaccuracy is going to affect the performance of the mechanism. Thus, the aggregate estimated error $\left(E_{m}\right)$ is a combination of the partial derivatives of the error function $F$ with respect to the individual independent parameters, as given by

$E_{m}=-\sum_{i=1}^{n} \frac{\partial f / \partial l_{i}}{\partial f / \partial \theta_{3}} \Delta l_{i}$

This total error will cause a deviation in output link angle as,

$E_{m}=\Delta \theta_{3}$

Re-arranging Eq. (7), the deviation at the output link, i.e., mechanical error in the mechanism, is estimated as:

$D \sin \theta_{3}+E \cos \theta_{3}=F$

$D=2 l_{1} l_{3} \sin \theta_{1}$

$E=2 l_{3} l_{4}+2 l_{1} l_{3} \cos \theta_{1}$

$F=2 l_{1} l_{4} \cos \theta_{1}+l_{1}^{2}-l_{2}^{2}+l_{3}^{2}+l_{4}^{2}$

Deviation $\Delta l$ in the link lengths $l_{1}, l_{2}, l_{3}$ and $l_{4}$ will modify the coefficients $D, E$, and $F$ by $\Delta D, \Delta E$ and $\Delta F$, respectively. Deviation in each link $i\left(\Delta l_{i}\right)$ will produce separate errors in $D, E$, and $F$, governed by an error $\Delta \theta_{3}$ in the output. The total mechanical error of the linkage $\left(E_{m}\right)$ will be the sum of the separate errors.

Error due to deviations $\Delta l_{1}, \Delta l_{2}, \Delta l_{3}$ and $\Delta l_{4}$ will be given as

$E_{m 1}=2 \frac{l_{4} \cos \theta_{1}+l_{1}-l_{3} \cos \left(\theta_{1}-\theta_{3}\right)}{D \cos \theta_{3}-E \sin \theta_{3}} \Delta l_{1}$

$E_{m 2}=\frac{-2 l_{2}}{D \cos \theta_{3}-E \sin \theta_{3}} \Delta l_{2}$

$E_{m 3}=-2 \frac{l_{4} \cos \theta_{1}+l_{3}-l_{1} \cos \theta_{3}}{D \cos \theta_{3}-E \sin \theta_{3}} \Delta l_{3}$

$E_{m 4}=2 \frac{l_{1} \cos \theta_{1}+l_{4}-l_{3} \cos \theta_{3}}{D \cos \theta_{3}-E \sin \theta_{3}} \Delta l_{4}$

The maximum and root-mean-square error, respectively, are given as

$E_{\max }=\left|E_{m 1}\right|+\left|E_{m 2}\right|+\left|E_{m 3}\right|+\left|E_{m 4}\right|$

$E_{\text {Total }}=E_{m 1}+E_{m 2}+E_{m 3}+E_{m 4}$

The above equations are used to determine the mechanical error for 4R and P3R mechanism due to link error [41].

\section{Kinematic synthesis of $4 R$ and P3R mechanism-exact estimation approach}

\subsection{Characteristic equation-4R mechanism}

Consider a four-bar mechanism as shown in Fig. 4, with $\mathrm{O}_{2}$ and $O_{4}$ as fixed pivots, the input to link 1 is $\theta_{1}$. The output angle $\theta_{3}$ can be found from component $\theta_{31}$ and $\theta_{32}$. Consider triangles $\mathrm{O}_{2} \mathrm{O}_{4} \mathrm{~A}$ and $\mathrm{AO}_{4} \mathrm{~B}$, with distance between $\mathrm{A}$ and $\mathrm{O}_{4}$ as $l_{\mathrm{r}}$ such that

$l_{r}^{2}=l_{1}^{2}+l_{4}^{2}-2 l_{1} l_{4} \cos (\psi)$

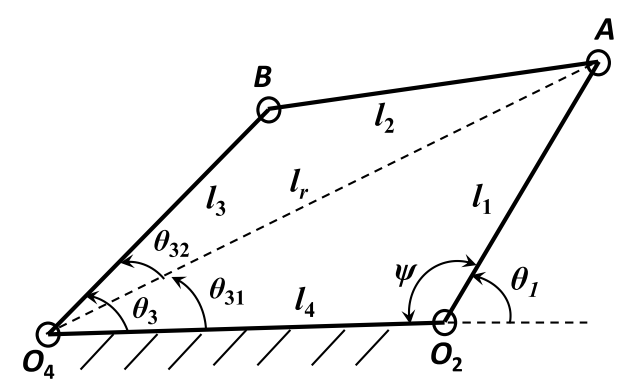

Fig. 4 Geometric analysis of $4 \mathrm{R}$ mechanism 
$l_{r}=\sqrt{l_{1}^{2}+l_{4}^{2}-2 l_{1} l_{4} \cos (\psi)}$

The output angle $\theta_{4}$ can be written as,

$\theta_{3}=\theta_{31}+\theta_{32}$

where,

$\theta_{31}=a \cos \left(\frac{l_{r}^{2}+l_{4}^{2}-l_{1}^{2}}{2 l_{r} l_{4}}\right)$

and

$\theta_{32}=a \cos \left(\frac{l_{r}^{2}+l_{3}^{2}-l_{2}^{2}}{2 l_{r} l_{3}}\right)$

Similarly, considering tolerances $\Delta l_{1}, \Delta l_{2}, \Delta l_{3}$ and $\Delta l_{4}$ on links $l_{1}, l_{2}, l_{3}$, and $l_{4}$, respectively, as shown in Fig. 5, the above equations can be rewritten as:

$l_{t 1}=l_{1}+\Delta l_{1}, l_{t 2}=l_{2}+\Delta l_{2}, l_{t 1}=l_{3}+\Delta l_{3}$ and $l_{t 4}=l_{4}+\Delta l_{4}$

$l_{t r}^{2}=l_{t 1}^{2}+l_{t 4}^{2}-2 l_{t 1} l_{t 4} \cos (\psi)$

$l_{t r}=\sqrt{l_{t 1}^{2}+l_{t 4}^{2}-2 l_{t 1} l_{t 4} \cos (\psi)}$

$\left(\theta_{31}\right)_{t}=a \cos \left(\frac{l_{t r}^{2}+l_{t 4}^{2}-l_{t 1}^{2}}{2 l_{t r} l_{t 4}}\right)$

$\left(\theta_{32}\right)_{t}=a \cos \left(\frac{l_{t r}^{2}+l_{t 3}^{2}-l_{t 2}^{2}}{2 l_{t r} l_{t 3}}\right)$

$\left(\theta_{3}\right)_{t}=\left(\theta_{31}\right)_{t}+\left(\theta_{32}\right)_{t}$
Equation (38) is used to obtain the output angle of the $4 \mathrm{R}$ mechanism with tolerances by the exact estimation approach.

\subsection{Characteristic equation-P3R mechanism}

Considering the $\mathrm{P} 3 \mathrm{R}$ configurations with tolerances $\Delta l_{1}, \Delta l_{2}$, $\Delta l_{3}$ and $\Delta l_{4}$ on the links $l_{1}, l_{2}, l_{3}$, and $l_{4}$, respectively, the link lengths are given as

$l_{t 1}=l_{1}+\Delta l_{1}, l_{t 2}=l_{2}+\Delta l_{2}, l_{t 1}=l_{3}+\Delta l_{3}$ and $l_{t 4}=l_{4}+\Delta l_{4}$

The modified mechanism is as shown in Fig. 6. The instantaneous leg length $v_{t}$ will be given as-

$v_{t}=\left(l_{t 1}+l_{t 2}\right)+\delta$

$(\psi)_{t}=a \cos \left(\frac{\left(v_{t}\right)^{2}+\left(l_{t 4}\right)^{2}-\left(l_{t 3}\right)^{2}}{2\left(l_{t 1}+\left(l_{t 2}+\delta\right)\right) l_{t 4}}\right)$

$\left(\theta_{3}\right)_{t}=a \cos \left(\frac{l_{t 4}^{2}+l_{t 3}^{2}-\left(v_{t}\right)^{2}}{2 l_{t 3} l_{t 4}}\right)$

$\left(\theta_{1}\right)_{t}=180-(\psi)_{t}$

\subsection{Mechanical error estimation-exact estimation approach}

The geometrical formulation for position analysis of mechanism forms a part of-exact estimation approach. To find the error at individual link, single link is considered to have tolerance at a time; and other links without tolerance. Error due to deviation on link 1, 2, 3 and 4, respectively, with tolerance conditions is given as under:

Error due to link 1,

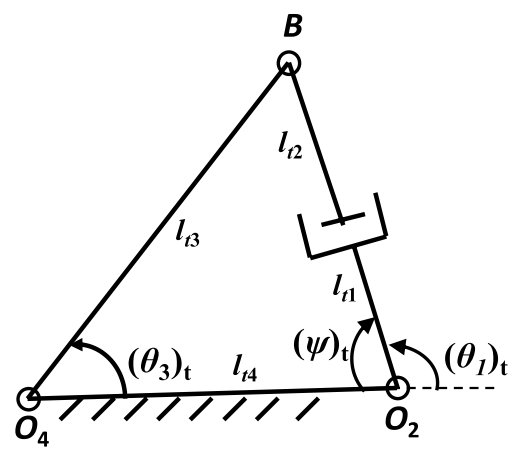

Fig. 6 P3R mechanism with link tolerances 
$l_{t 1}=l_{1}+\Delta l_{1}, l_{t 2}=l_{2}, l_{t 1}=l_{3}$ and $l_{t 4}=l_{4}$

$E_{d 1}=\left(\theta_{3}\right)-\left(\theta_{3}\right)_{t}$

Errors due to deviation on link 2:

$l_{t 1}=l_{1}, l_{t 2}=l_{2}+\Delta l_{2}, l_{t 1}=l_{3}$ and $l_{t 4}=l_{4}$

$E_{d 2}=\left(\theta_{3}\right)-\left(\theta_{3}\right)_{t}$

Errors due to deviation on link 3:

$l_{t 1}=l_{1}, l_{t 2}=l_{2}, l_{t 1}=l_{3}+\Delta l_{3}$ and $l_{t 4}=l_{4}$

$E_{d 3}=\left(\theta_{3}\right)-\left(\theta_{3}\right)_{t}$

Errors due to deviation on link 4:

$l_{t 1}=l_{1}, l_{t 2}=l_{2}, l_{t 1}=l_{3}$ and $l_{t 4}=l_{4}+\Delta l_{4}$

$E_{d 4}=\left(\theta_{3}\right)-\left(\theta_{3}\right)_{t}$

Combined error due to deviation on all links:

$l_{t 1}=l_{1}+\Delta l_{1}, l_{t 2}=l_{2}+\Delta l_{2}, l_{t 3}=l_{3}+\Delta l_{3}$ and $l_{t 4}=l_{4}+\Delta l_{4}$

$\left(E_{\text {Total }}\right)_{d}=E_{d 1}+E_{d 2}+E_{d 3}+E_{d 4}$

$\left(E_{\max }\right)_{d}=\left|E_{d 1}\right|+\left|E_{d 2}\right|+\left|E_{d 3}\right|+\left|E_{d 4}\right|$

\section{Case study-generalized proportions and identical operating conditions}

The mechanical error in four-bar 4R and P3R mechanisms of a given configuration is a function of tolerances on link dimensions and actuator input [1]. Both mechanisms are subjected to equivalent output generation conditions, and the required inputs are calculated.

\subsection{Equivalent condition between $4 R$ and P3R mechanism using analytical approach}

Figure $7 \mathrm{a}-\mathrm{b}$ shows the graphical representation of $4 \mathrm{R}$ and P3R mechanisms. Link 1 in $4 R$ and P3R mechanisms is given rotary and linear inputs, respectively. Link 3 generates a common path at output for both mechanisms, i.e., both mechanisms are subjected to identical operating conditions as seen in Fig. 7c-d. Link 3 and 4 are identical links (output and fixed link) in both mechanisms. Figure $7 b-d$ shows initial and final condition of P3R

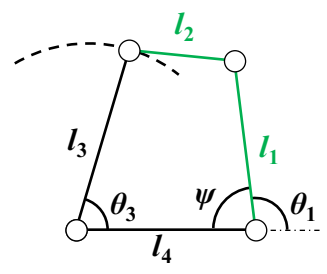

(a)

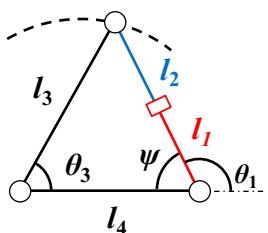

(b)

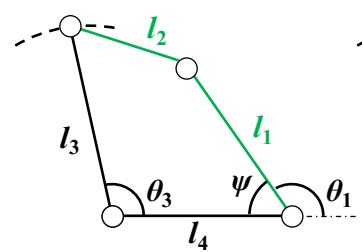

(c)

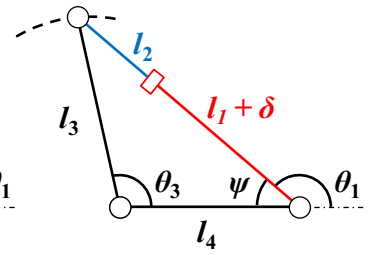

(d)
Fig. 7 4R (a, c) and P3R (b, d) mechanisms under identical operating conditions

mechanism with zero and maximum input displacement, respectively; Fig. $7 \mathrm{a}-\mathrm{c}$ represents equivalent $4 \mathrm{R}$ mechanism for output identical to P3R mechanism. For minimum and maximum linear displacement $(\delta)$ in $\mathrm{P} 3 \mathrm{R}$ mechanism, the range of angular displacement $\left(\theta_{1}\right)$ for $4 \mathrm{R}$ mechanism is obtained.

In Fig. $7 \mathrm{~d}$, the value of input link angle $\theta_{1}$ and output link angle $\theta_{3}$ is obtained by Eqs. (14) and (15), respectively. Substituting the value of $A, B$ and $C$ in Eq. (7), and rearranging the expressions as in Appendix-I,

$\sin \theta_{1} \cdot \sin \theta_{3}+\left(\frac{l_{4}}{l_{1}}+\cos \theta_{1}\right) \cdot \cos \theta_{3}=\frac{l_{4}}{l_{3}} \cos \theta_{1}+\frac{l_{1}^{2}-l_{2}^{2}+l_{3}^{2}+l_{4}^{2}}{2 l_{1} l_{3}}$

The final expression is,

$G \cdot \sin \theta_{1}+H \cdot \cos \theta_{1}=I$

where,

$G=\sin \theta_{3}$

$H=\cos \theta_{3}-\frac{l_{4}}{l_{3}}$

$I=\frac{l_{1}^{2}-l_{2}^{2}+l_{3}^{2}+l_{4}^{2}}{2 l_{1} l_{3}}-\frac{l_{4}}{l_{1}} \cdot \cos \theta_{3}$

$\theta_{1}=2 \cdot a \tan \left(\frac{G \pm \sqrt{G^{2}+H^{2}-I^{2}}}{H+I}\right)$

This equation provides input angle of $4 \mathrm{R}$ mechanism. 


\subsection{P3R mechanism proportions}

Effect of link proportions on behavior of the P3R and 4R mechanism is important for generality in applying formulation given in above sections. Initial P3R configuration is devised with the link proportions expressed in terms of link length $l_{4}$ as-

$l_{3}=1.5 \times l_{4}$

$l_{1}=l_{2}=\delta=\left(K \times l_{3}\right) / 3$

whereas $K$ is proportion factor within limiting constraint of $\mathrm{P} 3 \mathrm{R}$ configuration, such that.

$0.6<K<1.5$

On using $K$, desired variation in configuration is obtained. Amongst these proportions, selecting any sample proportion factor $K$ for P3R configuration, say 0.9 and assuming fixed link $l_{4}$ as 100 units, the link proportions can be given as

$l_{3}=1.5 \times l_{4}=150$;

$l_{1}=l_{2}=\delta=\left(K \times l_{3}\right) / 3=45$;

For all values of $K$, a set of dimensions is generated for further analysis.

\subsection{R mechanism proportions}

Now, to have the equivalent mechanism, $4 \mathrm{R}$ configuration is obtained for each variation in P3R mechanism. Assigning the fixed link $l_{4}$ of identical length as in P3R and using $\nu=\left(l_{1}+l_{2}+\delta\right)$ of $\mathrm{P} 3 \mathrm{R}$ configuration, the proportions for $4 \mathrm{R}$ mechanism identified as-

$l_{3}=1.5 \times l_{4}$

$l_{1}=l_{2}=((v) / 0.9) / 2$

Hereby using division factor 0.9 , non-singular $4 \mathrm{R}$ configuration is ensured. Similarly, the link proportions for $4 \mathrm{R}$ mechanisms will be given as

$l_{4}=100$

$l_{3}=1.5 \times l_{4}=150$;

$l_{1}=l_{2}=((v) / 0.9) / 2=75$

The maximum linear displacement for the P3R mechanism is 45 for $K=0.9$. Taking intermediate values between 0 and 45 , the values of output angle $\theta_{3}$ are determined, which are then used to calculate input angle $\theta_{1}$ for the $4 \mathrm{R}$ mechanism. Thus, for given value of total linear displacement $(\nu)$ in a P3R mechanism, the input angle $\left(\theta_{1}\right)$ for a $4 \mathrm{R}$ mechanism can be determined under the same output generation condition. Subsequent coupler positions, $P 1-P 11$,

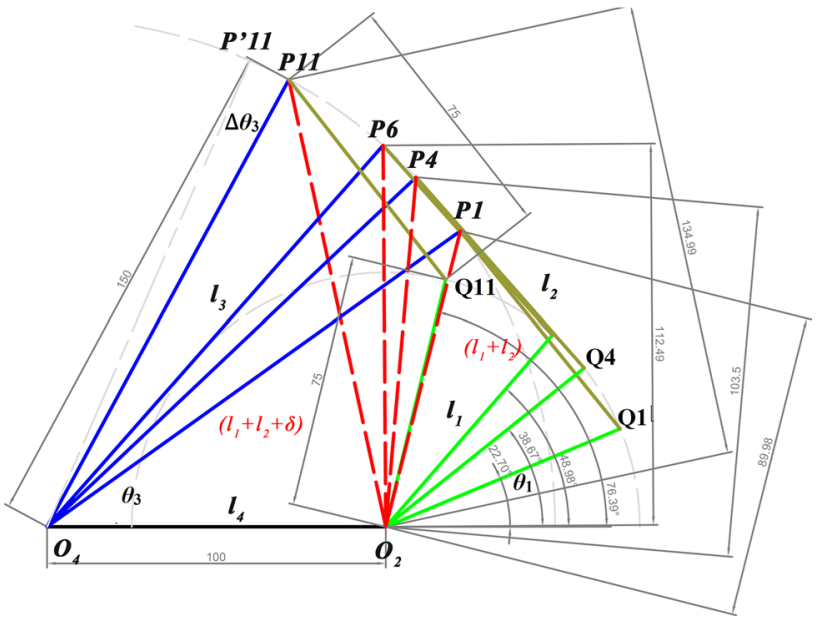

Fig. 8 CAD model for identical operating conditions of P3R and 4R mechanism

Table 1 Equivalent input conditions for P3R and 4R mechanism

\begin{tabular}{lll}
\hline $\begin{array}{l}\text { Linear Input Displace- } \\
\text { ment in P3R mechanism } \\
(\nu)\end{array}$ & $\begin{array}{l}\text { Position of } \\
\text { (common) } \\
\text { output }\end{array}$ & $\begin{array}{l}\text { Angular Input } \\
\text { Displacement in 4R } \\
\text { mechanism }\left(\theta_{1} \text { deg.) }\right.\end{array}$ \\
\hline 90 & $P 1$ & 22.7 \\
94.5 & $P 2$ & 28.16 \\
99 & $P 3$ & 33.46 \\
103.5 & $P 4$ & 38.67 \\
108 & $P 5$ & 43.83 \\
112.5 & $P 6$ & 48.98 \\
117 & $P 7$ & 54.17 \\
121.5 & $P 8$ & 59.43 \\
126 & $P 9$ & 64.83 \\
130.5 & $P 10$ & 70.45 \\
135 & $P 11$ & 76.39 \\
\hline
\end{tabular}

are obtained on account of the linear displacement of actuator. Therefore, an equivalence relation between input linear displacement and angular displacement for a specific output characteristic curve can be determined. The 4R mechanism in Fig. 8 is $\mathrm{O}_{2} Q 1 \mathrm{P}_{1} \mathrm{O}_{4}$ and equivalent P3R mechanism is $\mathrm{O}_{2} \mathrm{P}_{1} \mathrm{O}_{4}$. Length $\mathrm{O}_{2} \mathrm{P} 1$ indicates distance $(v)$ between the linear displacement of actuator $(\delta)$ and link length $\left(l_{1}+l_{2}\right)$ such that $v=l_{1}+l_{2}+\delta$. Similarly all other equivalent $\mathrm{P} 3 \mathrm{R}$ mechanisms are obtained for respective 4R mechanism from $P 1$ to $P 11$. The equivalent range of inputs in both mechanisms thus can be established as given in Table 1.

The equivalent input condition obtained for positions $P 1$ and $P 11$ by analytical approach is cross-checked using CAD modeling approach as seen in Fig. 8. 


\section{Results and discussion}

The mechanical error analysis of $4 \mathrm{R}$ and P3R mechanisms is carried out for a tolerance range. The notional values for range of tolerance, which could be obtained from manufacturing of the links, are considered for analysis. The estimated deviations are expressed as specific error, which is defined and applied herewith as the ratio of positional deviation and tolerance on links at that instance. This has made it possible to assess normalized tolerance effect in general.

\subsection{Effect of link proportion on error-P3R mechanism}

Error estimation of the P3R mechanism is carried out for various link proportions of the mechanism resulting from Eq. 56-57. The proportions of $l_{1}, l_{2}$ and $l_{3}$ are expressed in relation with fixed link $l_{4}$. For the proportion $l_{3}=1.5 \times l_{4}$ and displacement $\delta$ as $\left(K \times l_{3}\right) / 3$ varying between $0.6<K<1.5$, respectively, the error is estimated for individual link tolerances. The results are as expressed in Fig. 9a-d. Variation in error is observed for the increase in length of link 1 and link 2. For tolerance on individual links, fairly constant error for various positions is noticed at proportions with $K=0.9$. As link proportions shift toward the extreme values of output angle, the error values deviate away. The change in link proportions results in change in error pattern for individual link tolerances. The effect of tolerances on each individual link is estimated and represented.

\subsection{Effect of link proportion on error-4R mechanism}

Error estimation of the $4 \mathrm{R}$ mechanism is carried out for various link proportions of the mechanism equivalent to P3R mechanism, resulting from Eq. 58-59. The link 3 and link 4 have identical lengths as in P3R, and lengths of link 1 and link 2 are obtained for identical coupler position of P3R mechanism. The proportions of link 1, link 2 and link 3 are expressed in relation with fixed link $l_{4}$. Taking these link proportions for $4 \mathrm{R}$ mechanism, the error is estimated for individual link tolerances. The results are as expressed in

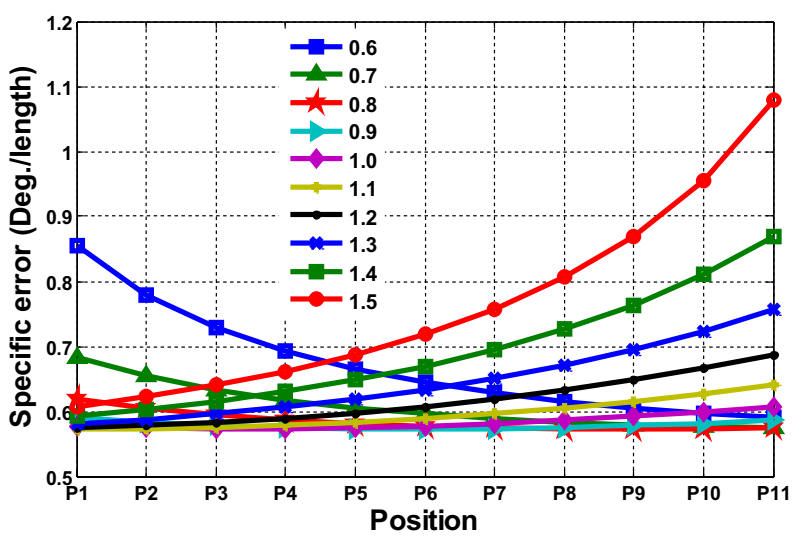

(a) Error pattern on Link - 1

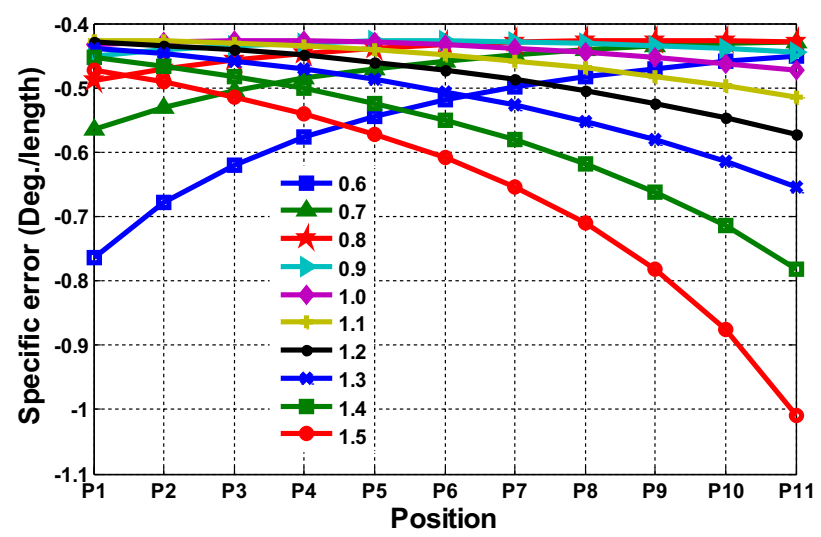

(c) Error pattern on Link - 3

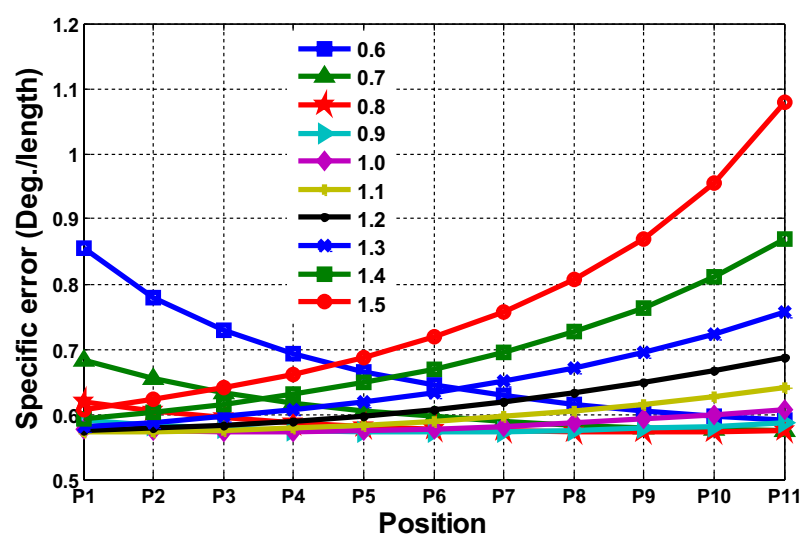

(b) Error pattern on Link - 2

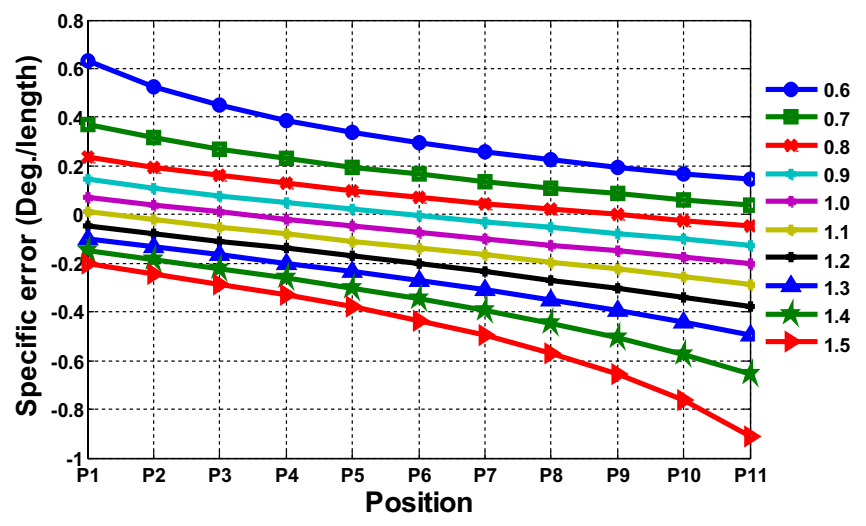

(d) Error pattern on Link - 4

Fig. 9 (a-d). Error estimations due to change in link proportions-P3R Mechanism 


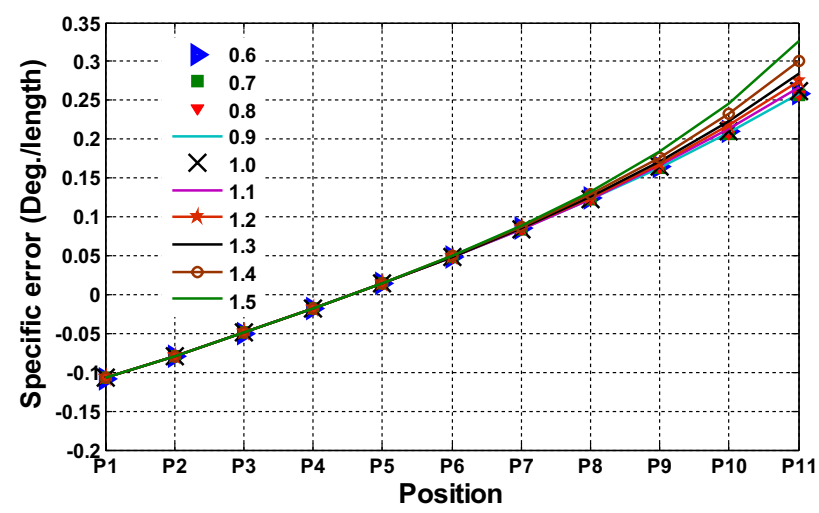

(a) Error pattern on Link - 1

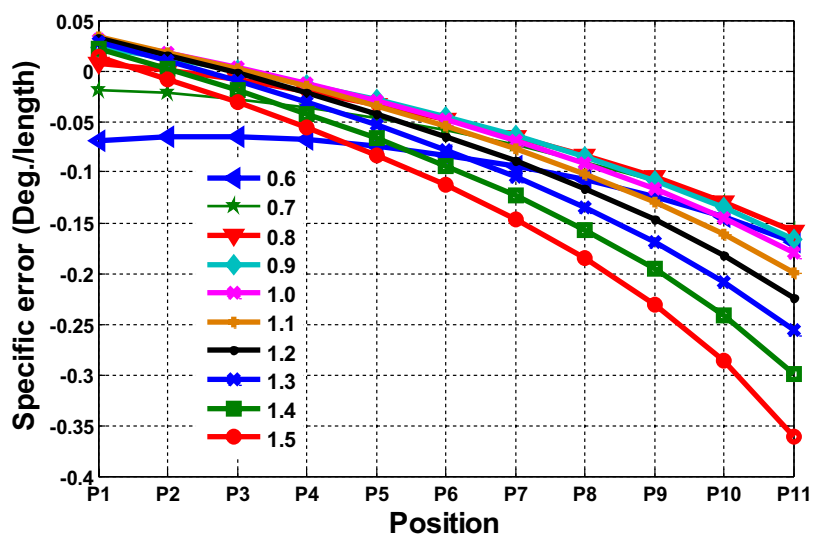

(c) Error pattern on Link - 3

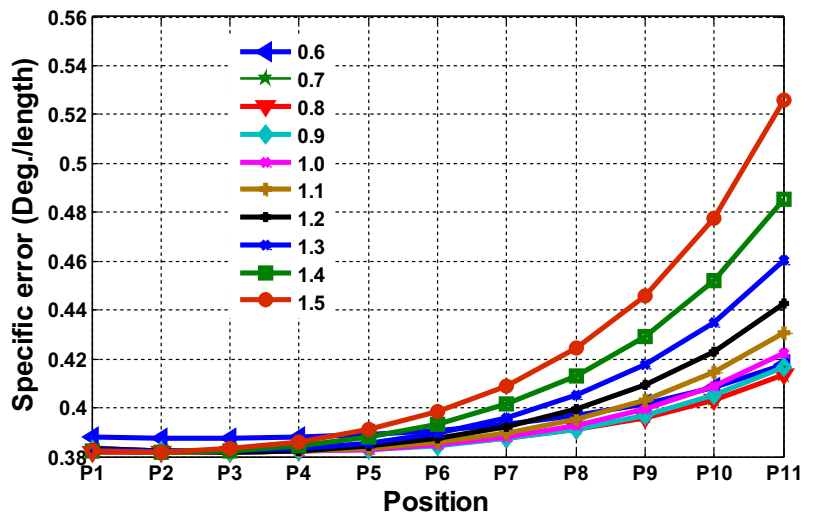

(b) Error pattern on Link - 2

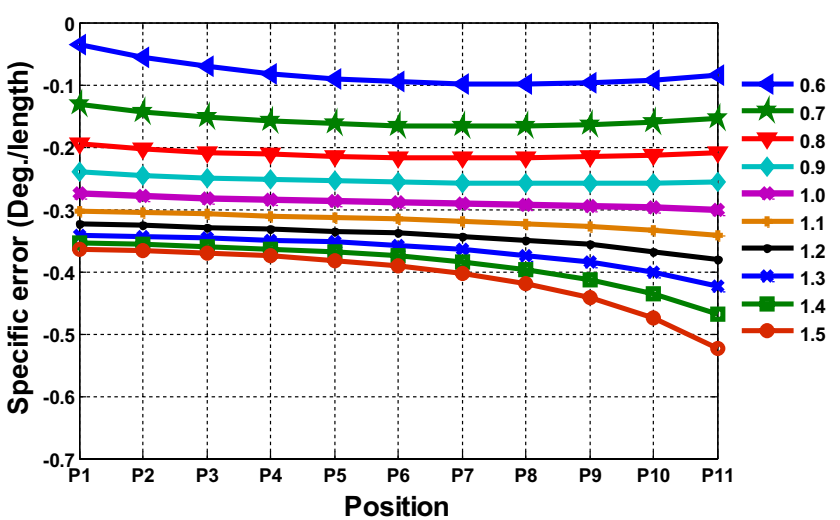

(d) Error patternon Link - 4

Fig. 10 (a-d) Error estimations due to change in link proportions-4R Mechanism

Fig. 10a-d. The convergence of the error pattern is seen for link-1 error. Variation in error is observed for the change in length of link 2. For tolerance on any link, fairly uniform error for various positions is noticed at proportions corresponding to $K=0.9$. As link proportions shift toward the minimum and maximum output angle in 4R configuration, the error values deviate away. The variation in link proportions results in change of error pattern for individual link tolerances.

The individualized error pattern is noted for respective variation in link proportion $(K)$ of links in $\mathrm{P} 3 \mathrm{R}$ and $4 \mathrm{R}$ mechanism and the uniform generalized estimations are not obtained. The subsequent error estimations are presented herewith for the proportion which presents approximately uniform error over various coupler positions, i.e., $K=0.9$.

\subsection{Errors in P3R mechanism}

Considering tolerance on link 1, link 2, link 3 and link 4, respectively, the specific error is estimated for P3R mechanism for any link proportions ( $K=0.6$ to 1.5 ) as represented

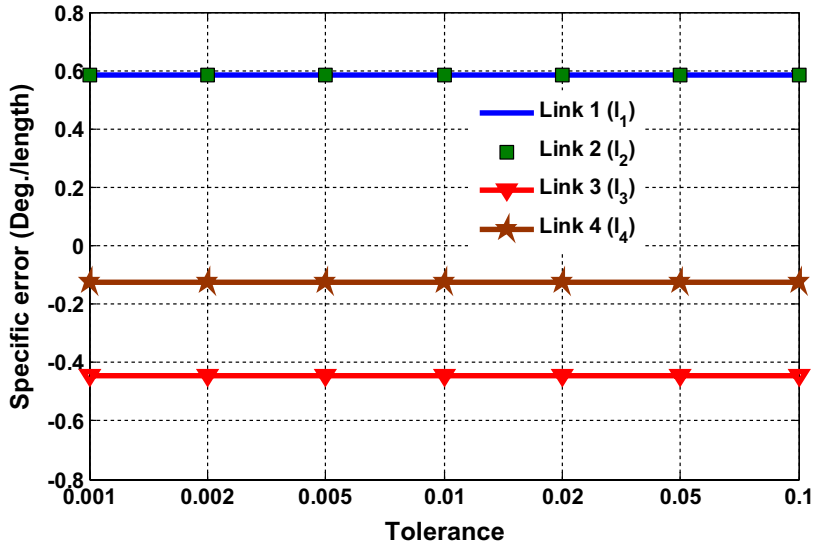

Fig. 11 Specific error of P3R mechanism with tolerance

in Fig. 11. Same results are observed for any set of link proportions and any coupler position.

The estimation against change in positions $\mathrm{P} 1$ to $\mathrm{P} 11$, the specific error due to tolerances on links 1 and 2 is found to be identical and above zero line, represented as overlapped 


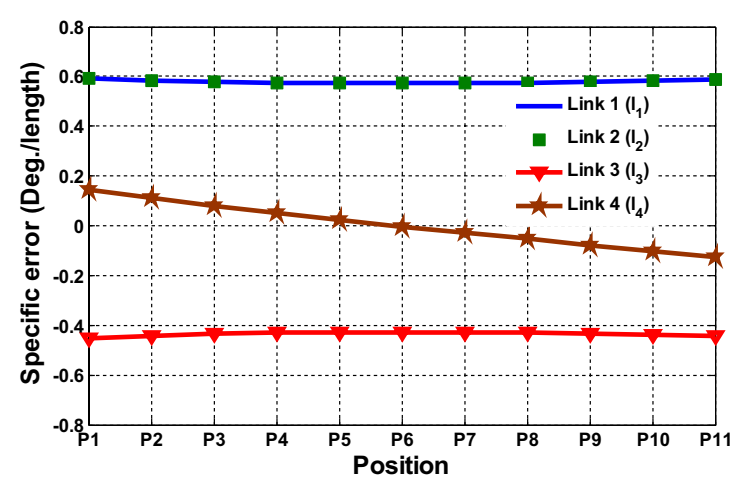

(a) Approximation approach

Fig. 12 Specific error of P3R mechanism with positions

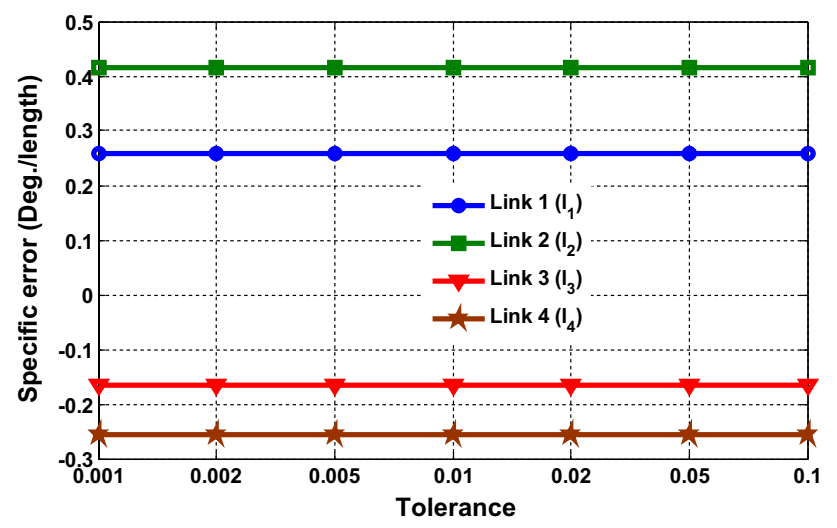

Fig. 13 Influence of links tolerances on specific error

lines. However, for the links 3 and 4 , it is below zero line with little difference in the magnitude. The effect of link tolerance on specific error at any tolerance value over coupler position is as represented in Fig. 12a, b.

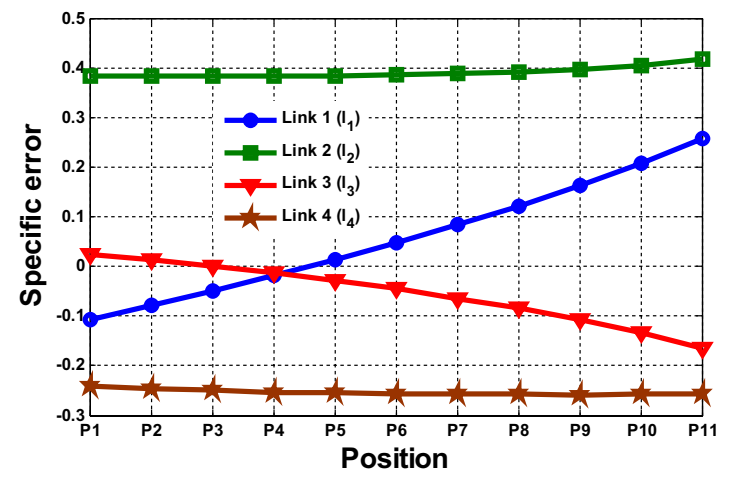

(a) Approximation approach

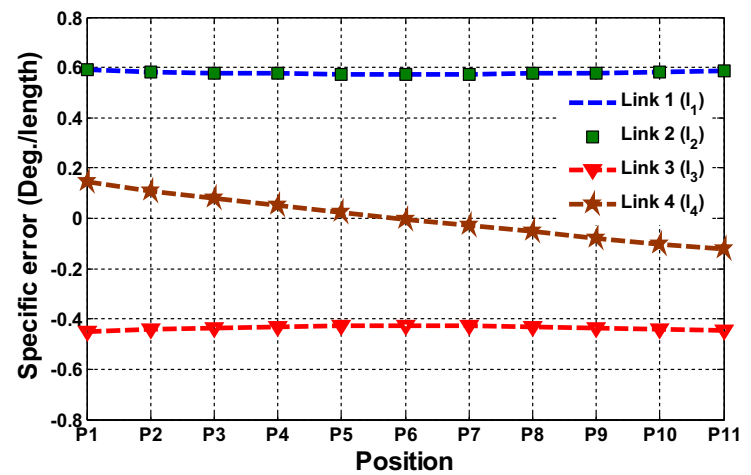

(b) Exact estimationapproach

\subsection{Error estimations in 4R mechanism- approximation and exact estimation approach}

The effect of increment in link tolerance on specific mechanical error is represented in Fig. 13. The specific error shows linear variation throughout the tolerance change. For any position of output link (i.e., $P 1$ to $P 11$ ) and any link proportions ( $K=0.6$ to 1.5 ), the specific error is uniform and constant for equivalent $4 \mathrm{R}$ mechanism.

The error deviation with respect to output link position is shown in Fig. 14(a-b). The specific error due to tolerances on links 1,2, 3 and 4 is a function of the input link orientation for achieving positions $P 1$ to $P 11$.

The result with approximation approach, i.e., Fig. 14a is verified through geometric formulation of exact estimation approach, as seen in Fig. 14b.

The change in tolerance sign (i.e., -0.001 to -0.1 ) results in reversing the error pattern for all links in both mechanisms, with sign reversed; without any change in magnitude of values and position [40].

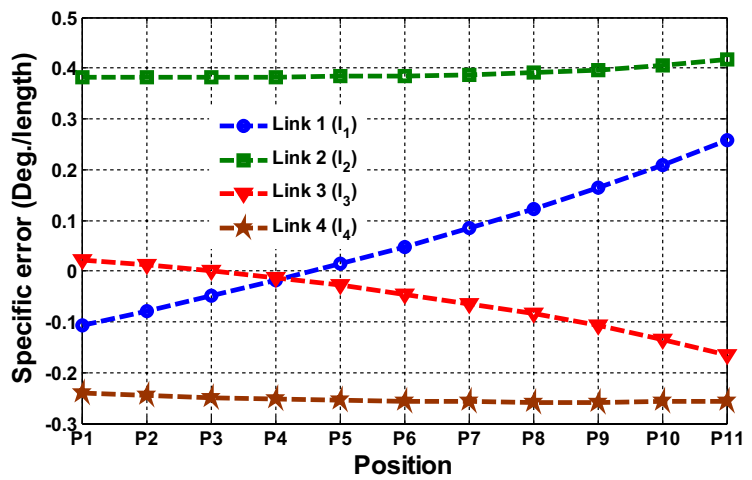

(b) Exact estimation approach

Fig. 14 Specific error of $4 \mathrm{R}$ mechanism with positions 


\subsection{Validation of estimations}

The estimations form the approximation and exact estimation approach is carried out as given in above sections. The estimations through the exact and approximation are giving most identical and conforming values. One of the estimations is presented below for P3R as well as $4 \mathrm{R}$ mechanisms, for link proportions with $K=0.9$, at position P6, and tolerance of 0.1 . The corresponding values are given in Table 2, showing the conformance. The same is also found by using CAD approach with the use of 2D drafting tool. The CAD estimation plotted with and without tolerances is shown in Fig. 15a, b. The estimations are conforming up to three decimal places.

Similarly, one of the estimation values for P3R mechanism is given in Table 3. The displacement $\delta$ of the actuator is 22.5 units, with link lengths $l_{1}$ and $l_{2}$ each 45 units as shown in Fig. 16a, b.

\section{Comparative error analysis in 4R and P3R mechanism}

Figure $17 \mathrm{a}, \mathrm{b}$ shows the comparison between $4 \mathrm{R}$ and $\mathrm{P} 3 \mathrm{R}$ mechanisms under the effect of link tolerance. The plot represents error deviation against coupler position and as well as link proportions. The maximum error in P3R mechanism

Table 2 Specific error estimation for $4 \mathrm{R}$ mechanism by CAD approach

Total error at $K=0.9$, Position $=(P 6)$, tolerance $(t)=0.1$

Approximation approach Exact estimation approach CAD approach

0.1308

0.130

0.130

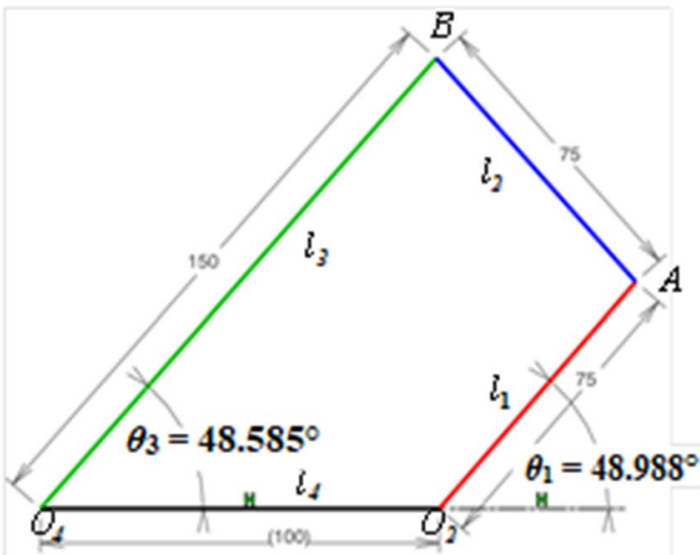

(a) Without tolerance
Table 3 Specific error estimation for P3R mechanism by CAD approach

Total error at $K=0.9$, Position $=(P 6)$, tolerance $(t)=0.1$

Approximation approach Exact estimation approach CAD approach

$\begin{array}{lll}0.7148 & 0.714 & 0.710\end{array}$

is higher than that in $4 \mathrm{R}$ mechanism. The maximum mechanical error is function of coupler position, as link proportion in P3R mechanism increases, the specific error reduces for coupler position $P 1$, reversing the findings toward position $P 11$. The error plot is fairly uniform for $4 \mathrm{R}$ mechanism, as seen in Fig. 17a. The total error bearing the compensatory effect of individual link errors represents the minimum and more realistic error representation, as in Fig. 17b. Identical error plots are obtained through geometrical analysis of exact estimation approach, thus verifying and validating the approximation approach results.

Thus, from performance point of view for identical output generation conditions at given tolerance, $4 \mathrm{R}$ mechanism is better in regards to mechanical error as compared to P3R mechanism.

\section{Conclusions}

The aim of the work carried out in this paper was to develop generalized formulation using modified Taylor series approximation method for positional error estimation in P3R and $4 \mathrm{R}$ planar manipulator configurations and subsequent validation of the approach using geometric approach and CAD model; followed by comparison of error in two configurations. This paper presents a detailed methodology for calculating mechanical errors in $4 \mathrm{R}$ and P3R mechanisms

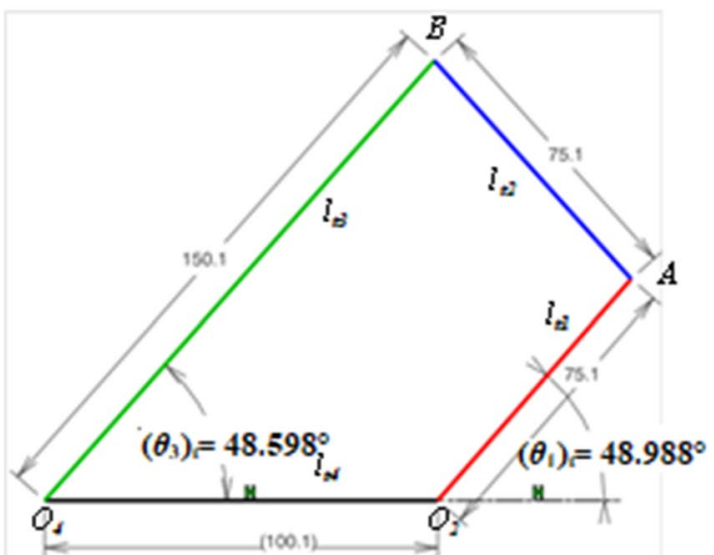

(b) With tolerance

Fig. 15 Specific error estimation for $4 \mathrm{R}$ mechanism by CAD approach 
Fig. 16 Specific error estimation for P3R mechanism by CAD approach

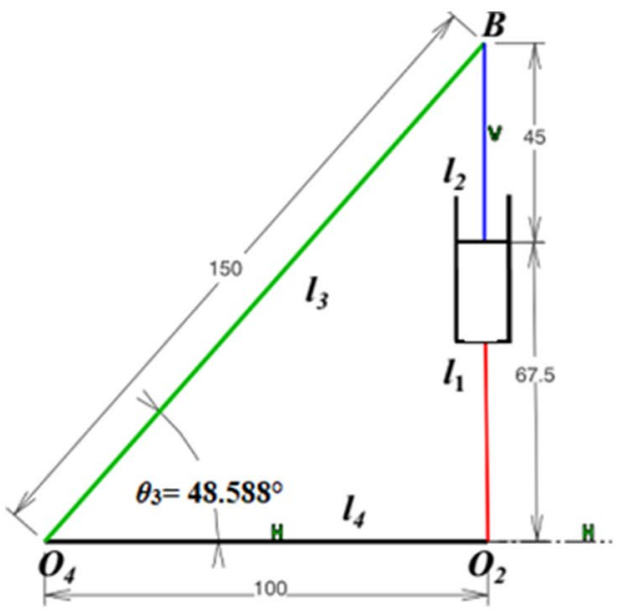

(a) Without tolerance

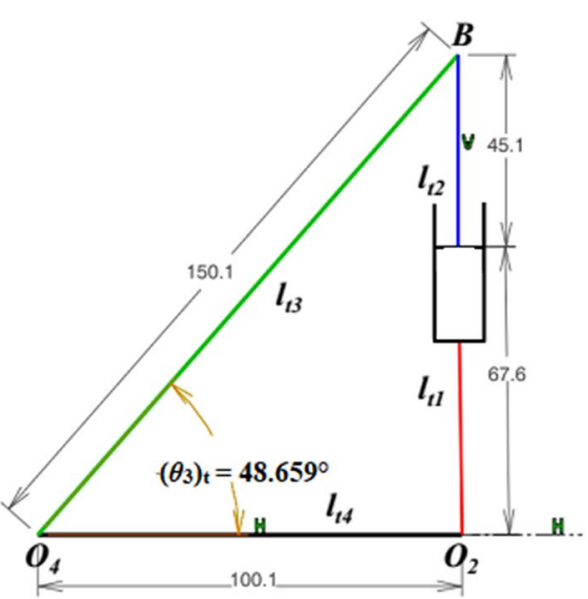

(b) With tolerance

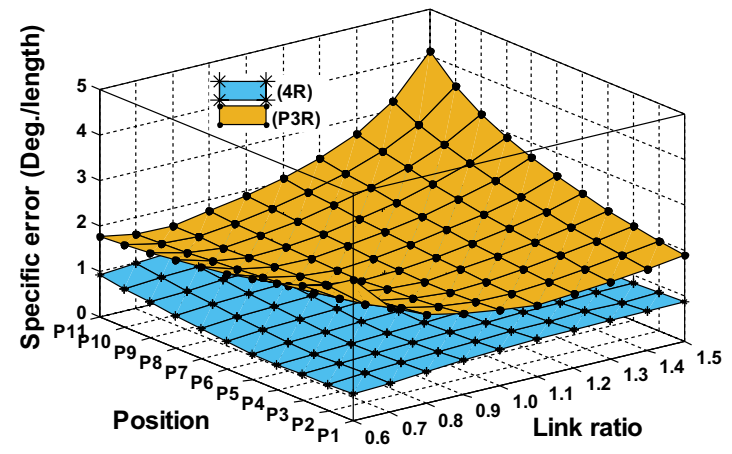

(a) Max error

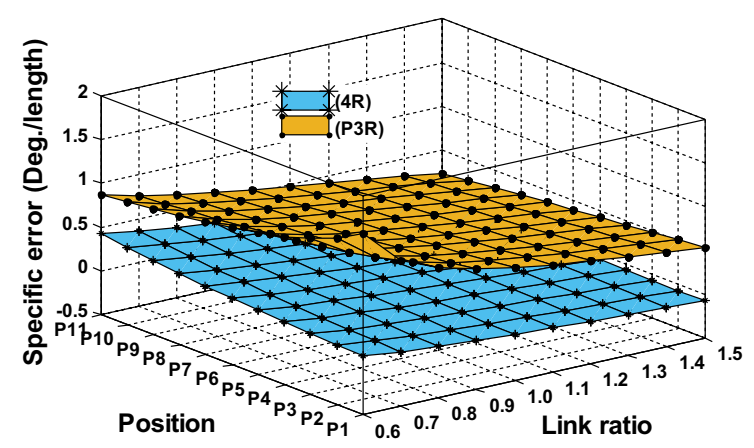

(b) Total error

Fig. 17 (a-d) Comparative error curve between P3R and 4R mechanism

by considering the influence of tolerances on the link dimensions. The formulation for mechanical error estimation in both configurations is developed. Traditional approximation approach is extended for analyzing mechanical error in P3R configuration. Error for individual link tolerance is estimated. The generality of applying the formulation is attempted, by analyzing various link proportions. The error variation pattern is investigated, leading to identification of a link ratio to have least error variation.

Geometric analysis for P3R and $4 \mathrm{R}$ configurations is devised as exact estimation approach; further used to verify and validate resulting error through approximation approach. Identical operating conditions are established between the two configurations. The methodology for the comparative evaluation of specific mechanical error in an active revolute and prismatic joint mechanism is presented. For the sample case of operational equivalence between these mechanisms, the method of error estimation is demonstrated. The estimated values are verified using CAD approach and generality of solution is checked.

Major contribution of the work lies in arriving at link proportions so as to have the minimal error, expressed as specific error, which shall help designer to select the link proportions.

The following generalized conclusions are drawn on the basis of this study:

1. The mechanical error varies from position to position throughout the range of operation i.e., positions P1 to P11 for both mechanisms. Traditionally, the error is suspected to have same over entire workspace.

2. The mechanical error depends on the link proportions. The link ratio can be identified to give least error variation pattern in $\mathrm{P} 3 \mathrm{R}$ and $4 \mathrm{R}$ configurations.

3. Comparative evaluation of mechanical error under link tolerance in $4 \mathrm{R}$ and $\mathrm{P} 3 \mathrm{R}$ mechanisms shows that $4 \mathrm{R}$ 
configuration is robust against P3R configuration under identical operating conditions. Thus, it is shown here, that revolute joint actuation becomes more advisable over prismatic actuation of robotic manipulators from positional error sensitivity perspective.

The established methodology and characterized performance will be useful in analyzing parallel mechanisms with rotary and linear actuation. The future scope lies in extending the approaches and methodology presented in this paper to study other issues related to mechanisms performance.

\section{Appendix I}

On substituting the values of $A, B$ and $C$ in Eq. (7) and rearranging the expressions,

$\sin \theta_{1} \cdot \sin \theta_{3}+\left(\frac{l_{4}}{l_{1}}+\cos \theta_{1}\right) \cdot \cos \theta_{3}=\frac{l_{4}}{l_{3}} \cos \theta_{1}+\frac{l_{1}^{2}-l_{2}^{2}+l_{3}^{2}+l_{4}^{2}}{2 l_{1} l_{3}}$

$\sin \theta_{1} \cdot \sin \theta_{3}+\frac{l_{4}}{l_{1}} \cdot \cos \theta_{3}+\cos \theta_{1} \cdot \cos \theta_{3}$

$-\frac{l_{4}}{l_{3}} \cos \theta_{1}-\frac{l_{1}^{2}-l_{2}^{2}+l_{3}^{2}+l_{4}^{2}}{2 l_{1} l_{3}}$

$\sin \theta_{1} \cdot \sin \theta_{3}+\cos \theta_{1} \cdot\left(\cos \theta_{3}-\frac{l_{4}}{l_{3}}\right)$

$=\frac{l_{1}^{2}-l_{2}^{2}+l_{3}^{2}+l_{4}^{2}}{2 l_{1} l_{3}}-\left(\frac{l_{4}}{l_{1}} \cdot \cos \theta_{3}\right)$

$G \cdot \sin \theta_{1}+H \cdot \cos \theta_{1}=I$

$G=\sin \theta_{3}$

$H=\cos \theta_{3}-\frac{l_{4}}{l_{3}}$

$I=\frac{l_{1}^{2}-l_{2}^{2}+l_{3}^{2}+l_{4}^{2}}{2 l_{1} l_{3}}-\frac{l_{4}}{l_{1}} \cdot \cos \theta_{3}$

Funding Open access funding provided by Manipal Academy of Higher Education, Manipal.

\section{Declarations}

Conflict of interest The author declares that he has no conflict of interest.
Open Access This article is licensed under a Creative Commons Attribution 4.0 International License, which permits use, sharing, adaptation, distribution and reproduction in any medium or format, as long as you give appropriate credit to the original author(s) and the source, provide a link to the Creative Commons licence, and indicate if changes were made. The images or other third party material in this article are included in the article's Creative Commons licence, unless indicated otherwise in a credit line to the material. If material is not included in the article's Creative Commons licence and your intended use is not permitted by statutory regulation or exceeds the permitted use, you will need to obtain permission directly from the copyright holder. To view a copy of this licence, visit http://creativecommons.org/licenses/by/4.0/.

\section{References}

1. Hartenberg R, Danavit J (1964) Kinematic synthesis of linkages. McGraw-Hill, New York

2. Jaiswal A, Jawale H (2017) Comparative study of four-bar hyperbolic function generation mechanism with four and five accuracy points. Arch Appl Mech 87:2037-2054

3. Choi J-H, Lee S-J, Choi D-H (1998) Stochastic linkage modeling for mechanical error analysis of planar mechanisms. J Struct Mech 26:257-276

4. Choubey M, Rao A (1982) Synthesizing linkages with minimal structural and mechanical error based upon tolerance allocation. Mech Mach Theory 17:91-97

5. Garrett R, Hall AS (1969) Effect of tolerance and clearance in linkage design. J Eng Ind 91:198-202

6. Kolhatkar S, Yajnik K (1970) The effects of play in the joints of a function-generating mechanism. J Mech 5:521-532

7. Erkaya S, Uzmay İ (2014) Modeling and simulation of joint clearance effects on mechanisms having rigid and flexible links. J Mech Sci Technol 28:2979-2986

8. Sharfi O, Smith M (1983) A simple method for the allocation of appropriate tolerances and clearances in linkage mechanisms. Mech Mach Theory 18:123-129

9. Ting K-L, Hsu K-L, Wang J (2017) Clearance-Induced Position Uncertainty of Planar Linkages and Parallel Manipulators. J Mech Robot 9:061001-061011

10. Flores $P$ (2011) A methodology for quantifying the kinematic position errors due to manufacturing and assembly tolerances. Strojniški vestnik-J Mech Eng 57:457-467

11. Ting K-L, Zhu J, Watkins D (2000) The effects of joint clearance on position and orientation deviation of linkages and manipulators. Mech Mach Theory 35:391-401

12. Dhande SG, Chakraborty J (1973) Analysis and synthesis of mechanical error in linkages-a stochastic approach. J Eng Ind 95:672-676

13. Mallik AK, Dhande SG (1987) Analysis and synthesis of mechanical error in path-generating linkages using a stochastic approach. Mech Mach Theory 22:115-123

14. Rhyu JH, Kwak BM (1988) Optimal stochastic design of fourbar mechanisms for tolerance and clearance. J Mech Transm Autom Des 110:255-262

15. Chakraborty J (1975) Synthesis of mechanical error in linkages. Mech Mach Theory 10:155-165

16. Ting K-L, Long Y (1996) Performance quality and tolerance sensitivity of mechanisms. J Mech Des 118:144-150

17. Erkaya S, Uzmay I (2009) Determining link parameters using genetic algorithm in mechanisms with joint clearance. Mech Mach Theory 44:222-234 
18. Zhu J, Ting K-L (2000) Uncertainty analysis of planar and spatial robots with joint clearances. Mech Mach Theory 35:1239-1256

19. Hafezipour M, Khodaygan S (2017) An uncertainty analysis method for error reduction in end-effector of spatial robots with joint clearances and link dimension deviations. Int J Comput Integr Manuf 30:653-663

20. Zhang X, Zhang X (2016) A comparative study of planar 3-RRR and 4-RRR mechanisms with joint clearances. Robot ComputIntegr Manuf 40:24-33

21. Chen G, Wang H, Lin Z (2013) A unified approach to the accuracy analysis of planar parallel manipulators both with input uncertainties and joint clearance. Mech Mach Theory 64:1-17

22. Jawale H, Thorat H (2013) Positional error estimation in serial link manipulator under joint clearances and backlash. J Mech Robot 5:021003

23. Flores $P$ (2010) A parametric study on the dynamic response of planar multibody systems with multiple clearance joints. Nonlinear Dyn 61:633-653

24. Flores P, Ambrósio J, Claro JCP, Lankarani H, Koshy C (2006) A study on dynamics of mechanical systems including joints with clearance and lubrication. Mech Mach Theory 41:247-261

25. Flores P, Ambrósio J, Claro JP (2004) Dynamic analysis for planar multibody mechanical systems with lubricated joints. Multibody SysDyn 12:47-74

26. Tian Q, Flores P, Lankarani HM (2018) A comprehensive survey of the analytical, numerical and experimental methodologies for dynamics of multibody mechanical systems with clearance or imperfect joints. Mech Mach Theory 122:1-57

27. Zhan Z, Zhang X, Jian Z, Zhang H (2018) Error modelling and motion reliability analysis of a planar parallel manipulator with multiple uncertainties. Mech Mach Theory 124:55-72

28. Erkaya S (2018) Effects of joint clearance on the motion accuracy of robotic manipulators. Strojniski Vestnik/J Mech Eng. https:// doi.org/10.5545/sv-jme.2017.4534

29. Cammarata A (2017) A novel method to determine position and orientation errors in clearance-affected overconstrained mechanisms. Mech Mach Theory 118:247-264

30. Erkaya S (2018) Clearance-induced vibration responses of mechanical systems: computational and experimental investigations. J Braz Soc Mech Sci Eng 40(2):1-12
31. Erkaya S (2013) Trajectory optimization of a walking mechanism having revolute joints with clearance using ANFIS approach. Nonlinear Dyn 71(1):75-91

32. Erkaya S, Uzmay I (2010) Experimental investigation of joint clearance effects on the dynamics of a slider-crank mechanism. Multibody Syst Dyn 24:81-102

33. Erkaya S, Uzmay I (2009) Investigation on effect of joint clearance on dynamics of four-bar mechanism. Nonlinear Dyn 58:179

34. Tsai M-J, Lai T-H (2008) Accuracy analysis of a multi-loop linkage with joint clearances. Mech Mach Theory 43:1141-1157

35. Jawale H, Thorat H (2014) Positional accuracy analysis in serial chain and four-bar closed chain manipulator. In: ASME 2014 international mechanical engineering congress and exposition, American society of mechanical engineers, pp. V011T014A010-V011T014A010

36. Li X, Ding X, Chirikjian GS (2015) Analysis of angular-error uncertainty in planar multiple-loop structures with joint clearances. Mech Mach Theory 91:69-85

37. Tsai M-J, Lai T-H (2004) Kinematic sensitivity analysis of linkage with joint clearance based on transmission quality. Mech Mach Theory 39:1189-1206

38. Wu W, Rao S (2004) Interval approach for the modeling of tolerances and clearances in mechanism analysis. J Mech Des 126:581-592

39. Zhang D, Han X (2020) Kinematic reliability analysis of robotic manipulator. J Mech Des. https://doi.org/10.1115/1.4044436

40. Jaiswal A, Jawale H (2016) Comparative study of mechanical error in P3R and 4R manipulators. In: ASME 2016 international design engineering technical conferences and computers and information in engineering conference, American society of mechanical engineers, pp. V05BT07A044-V005BT007A044

41. Jawale HP, Jaiswal A (2018) Investigation of mechanical error in four-bar mechanism under the effects of link tolerance. J Braz Soc Mech Sci Eng 40:383

Publisher's Note Springer Nature remains neutral with regard to jurisdictional claims in published maps and institutional affiliations. 